\section{P1-S2.13 THE IMPACT OF THE 2010 WINTER OLYMPIC GAMES ON SEX WORK PATTERNS, SAFETY AND SEX WORKER VULNERABILITY TO HIV AND SEXUALLY TRANSMITTED INFECTIONS}

doi:10.1136/sextrans-2011-050108.70

${ }^{1} \mathrm{~K}$ Deering, ${ }^{1} \mathrm{~J}$ Chettiar, ${ }^{2} \mathrm{~K}$ Chan, ${ }^{3} \mathrm{M}$ Taylor, ${ }^{1} \mathrm{~J}$ Montaner, ${ }^{1} \mathrm{~K}$ Shannon. ${ }^{1}$ University of British Columbia, Vancouver, Canada; ${ }^{2} B C$ Centre for Excellence in HIVIAIDS, Canada; ${ }^{3}$ HUSTLE, PEERS Vancouver Resource Society, Canada

Background Large-scale international sports events such as the 2010 Winter Olympics Games in Vancouver, Canada can have significant impacts on the vulnerability of sex workers (SWs) to HIV and sexually transmitted infections (STIs). SWs in Vancouver continue to face extreme vulnerabilities to violence and HIV/STIs. Despite significant focus on the social legacy of large-scale sports events, there is surprisingly limited research evaluating the impact of such events on sex work. This study therefore examined the impact of the 2010 Olympic time period on sex work patterns, safety and HIV/STI vulnerability of SWs in Vancouver.

Methods Data were used from a screening questionnaire for a longitudinal cohort study of 230 SWs from January to July 2010. Bivariate and multivariable logistic regression was used to examine the impact of time period (pre/during-Olympics vs post-Olympics) on sex work patterns, safety and HIV/STI vulnerability in the last 30 days.

Results Overall, the median age of respondents was 33 years (IOR-28-40), and 106 (51.2\%) SWs were non-Caucasian. In multivariable analysis (after adjusting for social and environmental factors) in the pre/during-Olympics period compared to postOlympics, we found significantly higher odds of respondents reporting more police stopping SWs without arrest (adjusted ORs [AOR]-3.95, 95\% CIs 1.92 to 8.14), reporting a decrease in the numbers of clients available (AOR-1.97, 95\% CI 1.11 to 3.48), reporting difficulty hooking up with clients due to road closures/ construction (AOR 7.68, 95\% CI 2.46 to 23.98) and a decrease in the numbers of clients available (AOR 3.59, 95\% CI 1.79 to 7.19 ). We found no significantly increased odds in new/trafficked SWs in the Olympic time period.

Conclusions There were significant changes in sex work patterns, safety and HIV/STI vulnerability of SWs immediately before and during the 2010 Winter Olympics compared to post-Olympics. Fears over an influx of new SWs or human trafficking appear to be unfounded. Displacement of SWs away from main streets/ commercial areas has significant public health implications, since this previously has been shown to promote violence and coercive unprotected sex, and increase risk for HIV/STIs. Safer sex work spaces such as indoor brothels and policy reforms should be considered both in Canada and by other host countries of large-scale events to reduce the vulnerability of SWs to HIV from displacement and disruption.

\section{P1-S2.14 NEISSERIA GONORRHOEA AND CHLAMYDIA TRACHOMATIS RE-INFECTION AND ASSOCIATED RISK FACTORS AMIONG COHORT OF FEMALE SEX WORKERS IN INDIA}

doi:10.1136/sextrans-2011-050108.71

${ }^{1} \mathrm{~A}$ R Risbud, ${ }^{1} \mathrm{G}$ R Deshpande, ${ }^{2} \mathrm{P}$ Narayanan, ${ }^{2} \mathrm{P}$ Parimi, ${ }^{2}$ Anjana Das. ${ }^{1}$ National AIDS Research Institute Pune, India; ${ }^{2}$ Family Health International, India. New Delhi, India

Background Neisseria gonorrhoea (NG) and Chlamydia trachomatis (CT) infections can be completely cured by appropriate medication. Syndromic STI management often fails to cover asymomatic NG/ CT infections. Additionally, Female sex workers (FSWs), due to their risky behaviour are vulnerable to repeated infections. Frequency of repeated infections with these pathogens was studied in a cohort of FSWs.

Methods In 6 months prospective cohort study, FSWs aged 18-40 years were recruited from three STI clinics in two states in India as a part of Bill \& Melinda Gates Foundation funded operational research. Vaginal swab specimens were collected in all visits and were tested for NG/CT by Gen-Probe APTIMA Combo II assay. Presumptive treatment for gonorrhoea and chlamydia at the baseline visit and syndromic STI management at all subsequent visits was provided during the study period. Re-infection was defined as a laboratory confirmed NG/CT infection occurring after 30 days from an initial confirmed positive test result.

Results Of 417 FSWs recruited during 2008-2009, 360 made at least 1-monthly follow-up visit. At baseline and during follow-up, 184 were NG infected and 210 were CT infected see Abstract P1S2.14 Table 1. A total of 39 (11.8\%) NG re-infections and 30 (8.3\%) CT re-infections were identified at follow-up visits. Among the factors studied, alcohol consumption $(\mathrm{OR}=2.4, \mathrm{p}=0.01)$; relatively new to commercial sex work (within 3 years) $(O R=2.16, p=0.03)$; had STIs in the past $(O R=3.85, p=0.00)$ were significantly associated with the NG, CT re-infection.

Conclusion High frequency of NG and CT re-infection among FSWs highlights the need for regular screening for etiological diagnosis for effective treatment. New sex workers and those consuming alcohol need special attention in STI management.

Abstract P1-S2.14 Table 1 Re-infection frequency among female sex workers Cohort in India

\begin{tabular}{lrrrrrr}
\hline NG-Re-infection & Visit 1 & Visit 2 & Visit 3 & Visit 4 & Visit 5 & Total \\
\hline No re-infection/Negatives & 417 & 343 & 288 & 244 & 175 & 1467 \\
Re-infected (31-330 days) & 00 & 17 & 4 & 6 & 12 & 39 \\
Total & 417 & 360 & 292 & 250 & 187 & 1506 \\
CT-Re-infection & & & & & & \\
No re-infection/ Negatives & 417 & 346 & 282 & 250 & 181 & 1476 \\
Re-infected (30-291 days) & 00 & 14 & 10 & 0 & 6 & 30 \\
Total & 417 & 360 & 292 & 250 & 187 & 1506 \\
\hline
\end{tabular}

\section{P1-S2.15 PREVALENCE OF CONDYLOMA ACUMINATUM AND CERTAIN SEXUALLY TRANSMITTED DISEASES AMONG FEMALE SEX WORKERS (FSWS) IN A COHORT STUDY AT SEXUALLY TRANSMITTED DISEASES DISPENSARY (DIST) IN COTONOU, BÉNIN}

doi:10.1136/sextrans-2011-050108.72

${ }^{1} \mathrm{~N}$ Nassirou, ${ }^{2} \mathrm{~S}$ Diabate, ${ }^{3} \mathrm{~J}$ Akakpo, ${ }^{1} \mathrm{C}$ Tremblay, ${ }^{4} \mathrm{M}$ Alary. ${ }^{1}$ Dispensaire IST, Centre de sante de Cotonou1; ${ }^{2}$ Centre de recherche du centre hospitalier de I'Université de Montréal, Montréal; ${ }^{3}$ CTA, Centre National hospitalier universitaire Hubert Koutoukou Maga de Cotonou, Cotonou, Benin; ${ }^{4}$ URESP, Centre de recherche FRSO du CHA université de Québec, Québec, Canada

Background Condyloma acuminatum usually diagnosed among FSWs, represents an entrance for HIV infection. This study aimed to determine the prevalence of condyloma acuminatum and factors associated with its appearance among FSWs who attended this STI dispensary.

Methods From September 2008 to March 2010, each FSWs of the cohort at recruitment was probed with a questionnaire on her sexual behaviours, social and demographic characteristics, to provide a blood sample for HIV screening, vaginal and cervix samples for 